\title{
CARACTERIZAÇÃO DE QUATRO GRUPOS DE JABUTICABEIRA, NAS CONDIÇÕES DE JABOTICABAL-SP ${ }^{1}$
}

\author{
NATANAEL DE JESUS ${ }^{2}$, ANTONIO BALDO GERALDO MARTINS ${ }^{3}$, EDUARDO JOSÉ DE ALMEIDA², JOSÉ BASÍLIO \\ VIEIRA LEITE ${ }^{4}$, RITA MARIA DEVÓS GANGA ${ }^{2}$, ERIVALDO JOSÉ SCALOPPI JUNIOR ${ }^{4}$, \\ RENATA APARECIDA DE ANDRADE ${ }^{4}$, RICARDO FRANCO CUNHA MOREIRA ${ }^{4}$
}

\begin{abstract}
RESUMO - O presente trabalho foi conduzido com o objetivo de avaliar as características morfológicas e físicas da planta e do fruto de jabuticabeiras, pertencentes à coleção da FCAV-UNESP, Câmpus de Jaboticabal-SP. A coleção conta com 160 plantas, das quais 40, que apresentavam frutificação no momento da seleção, foram agrupadas, preliminarmente, segundo características semelhantes, em quatro grupos denominados JAB 01, JAB 02, JAB 03 e JAB 04. Mediram-se nestes grupos a altura da planta, diâmetro do tronco a $20 \mathrm{~cm}$ do solo, diâmetro de copa, comprimento do entrenó e largura da folha. Os frutos, foram avaliados quanto a comprimento, largura, massa fresca da casca, da semente, da polpa e total, bem como o número de sementes por fruto. O delineamento utilizado foi o inteiramente casualisado, com 4 tratamentos, quatro grupos de plantas e 5 repetições, com 2 plantas por repetição. Os dados foram submetidos à análise de variância pelo teste $(F)$ e as médias comparadas pelo teste de Tukey. Observou-se grande variabilidade nos grupos estudados. O JAB-04 revelou-se como o mais promissor para utilização em programas de melhoramento, pela precocidade de produção, baixo porte e maior rendimento em polpa, apesar de os frutos apresentarem menor tamanho em relação aos dos demais grupos.
\end{abstract}

Termos para indexação: Myrciaria cauliflora, crescimento, fruto, caracterização morfológica

\section{CHARACTERIZATION OF FOUR GROUPS OF JABOTICABA TREES, IN CONDITIONS OF JABOTICABAL CITY, SÃO PAULO STATE, BRAZIL}

\begin{abstract}
The present research was carried out with the objective to evaluate the morphological and physical characteristics of plants and fruits of jaboticaba trees, from the collection of the FCAV - UNESP, Campus of Jaboticabal, São Paulo State. Among the 160 plants of the collection, 40 flowering trees were selected for this research. These plants were grouped according to morphological simmilarities, in four groups, identified as Jab1, Jab-2, Jab-3 and Jab-4. In these groups the folowing variables were evaluated: plant heigh, stalk diamenter, crows diameter, brauch internode lengh, leaf width and lengh, fruit diameter, fruit lengh, number of seeds per fruit and fresh mass of the bark, pulp and seed of the fruits. Data were statistically analised as a completed randomized plots design, with your treatments (four groups of plants) and five replications composed by two plants each. Analysis of variance was performed by (F) test, and the means were compared by Tuckey test. It was observed great variability among studied groups. The group Jab-4 was the most promising for breeding programs because its precocity, fruit production smaller trees larger proportion of pulp, in spite of smallen fruits size in comparison to these from other groups.
\end{abstract}

Index terms: Myrciaria cauliflora, development, fruit, morphologic characterization

\section{INTRODUÇÃO}

A jabuticabeira (Myrciaria cauliflora Berg.), frutífera pertencente à família Myrtaceae, é tipicamente brasileira (Ochse, 1966), do gênero Myrciaria, porém quanto à classificação há controvérsias (Pérez \& Martins, 2002). Para Barbosa (sd) citado por Gomes (1983), são duas as espécies mais importantes, a $M$. jaboticaba, com frutos pequenos de pedúnculo escuro e a $M$. cauliflora, com frutos grandes e sésseis. Já Bailey, 1920; Corrrea, 1969, e Benza, 1980, mencionam também a $M$. truncifolia; no entanto, das três, a espécies mais difundida no Brasil é a M. cauliflora (Gomes, 1983).

As variedades de jabuticabeira mais cultivadas são: a 'Paulista', de grande porte, alta capacidade de produção, frutos graúdos, e de maturação tardia; a 'Sabará', que é a mais apreciada e mais cultivada, cresce pouco, é precoce, e produz muitos frutos pequenos e doces; a 'Branca', de porte médio, com muitos frutos grandes, verde-claros; a 'Rajada', que se assemelha muito às duas primeiras em crescimento e produção, tem os frutos verde-bronzeados, grandes e doces, mas sua maturação é mediana, e a 'Ponhema', mais apropriada para a fabricação de geléias, doces e licores, apresenta maior crescimento, alta produção e com frutos grandes, que só devem ser consumidos quando bem maduros (Gomes, 1983).

A jabuticabeira apresenta copa com forma variada, porte médio a grande, com 6 a 9 m de altura, podendo alcançar até $12 \mathrm{~m}$ com tendência a abundante esgalhamento, tendo como características típicas o hábito de frutificação nos ramos e troncos com ruptura da casca (Benza, 1980; Andersen \& Andersen, 1989). Florescem mais de uma vez ao ano, com flores novas recobrindo a periferia dos ramos, dando-lhes característica ornamental. Seus ramos são delgados, cilíndricos e glabros; as folhas, opostas e elípticas, são incompletas, com bainha ausente e pecíolo presente. A lâmina foliar apresenta entre 2,4 e $4,3 \mathrm{~cm}$ de comprimento e 0,6 a 1,6 cm de largura (Donadio et al., 2002).

Wilbank et al. (1983) citam que o fruto é uma baga, subgloboso, negro quando maduro, liso, com 1,6 a 2,2 cm de diâmetro, contendo de 1 a 4 sementes. A casca é fina e muito frágil; a polpa é doce com leve acidez, de ótimo sabor e de cor branca a translúcida. Já Benza, (1980) relata que os frutos são globosos, atingindo até $3,5 \mathrm{~cm}$ de diâmetro, de polpa branca e sabor subácido com sementes variando de ovais a redondas e lateralmente compridas, podendo chegar a até 4 por fruto.

Conforme Donadio et al. (2002), todas as jabuticabeiras possuem sementes poliembriônicas, o número de embriões pode chegar a 5. Os cotilédones são avermelhados e bastante pequenos, com comprimento variando de 2 a $12 \mathrm{~mm}$ (Soubihe Sobrinho \& Gurgel, 1952).

O potencial de comercialização do fruto é grande, em função de suas características organolépticas para consumo ao natural, utilizado na fabricação de licores e geléias (Magalhães et al., 1996). Donadio (2000) relata que, além do consumo ao natural, o fruto pode ser utilizado para fabricação de xaropes, vinho, licor, vinagre e geléia, tendo grande potencial econômico, com significativo crescimento na sua comercialização que, em 1998, foi superior a 4 mil toneladas na Companhia de Entrepostos e Armazéns Gerais de São Paulo (CEAGESP).

Mesmo com esse grande potencial descrito, ainda existem poucos estudos sobre a caracterização das diferentes plantas desta frutífera, e por esta razão, são encontradas plantas com variados

\footnotetext{
${ }^{1}$ (Trabalho 171/2003). Recebido: 11/11/2003. Aceito para publicação: 17/11/2004.

${ }^{2}$ Mestrandos em Agronomia - UNESP- FCAV - Câmpus de Jaboticabal - Depto de Produção Vegetal. Via de Acesso Prof. Paulo Donato Castellane, s/n. Cep: 14884900. Jaboticabal-SP. Tel/Fax: (16) 3209-26668. e-mail: natanael@ fcav.unesp.br

${ }^{3}$ Eng. Agr., Prof. Doutor - UNESP - FCAV - Depto de Produção Vegetal. e -mail: baldo@ fcav.unesp.br

${ }^{4}$ Doutorandos em Agronomia - UNESP- FCAV - Câmpus de Jaboticabal -
}

Rev. Bras. Frutic., Jaboticabal - SP, v. 26, n. 3, p. 482-485, Dezembro 2004 
comportamentos fenotípicos, de produção e qualidade dos frutos em bosques comercias explorados com a cultura. Tanto é que Pereira et al. (2000), avaliando atributos físicos e químicos de oito clones de jabuticabeira, verificaram que, dentre os clones estudados, havia uma grande variação no formato do fruto, e mesmo a 'Sabará' (a mais difundida), não foi possível afirmar que sua forma esférica encontrada seja determinante.

Desta forma, objetivou-se avaliar características morfológicas de plantas e de frutos de jabuticabeiras pertencentes aos grupos da coleção da FCAV, Câmpus de Jaboticabal-SP, com o intuito de verificar quais seriam mais promissores em programas de melhoramento.

\section{MATERIAL E MÉTODOS}

As avaliações foram realizadas em jabuticabeiras com 25 anos, de livre crescimento, conduzidas sem irrigação ou adubação e em espaçamento de $6 \times 6 \mathrm{~m}$, pertencentes à coleção da Faculdade de Ciências Agrárias e Veterinárias (FCAV/UNESP), Câmpus de Jaboticabal-SP, no período de setembro a outubro de 2002. As coordenadas geográficas do local são latitude $21^{0} 17^{\prime} 05^{\prime}$ 'S e longitude $48^{\circ} 17^{\prime} 09^{\prime \prime} \mathrm{W}$, com altitude de aproximadamente $590 \mathrm{~m}$. O clima da região, segundo classificação de Köeppen é do tipo Cwa, subtropical, relativamente seco no inverno, com chuvas no verão, apresentando temperatura média anual de $22^{\circ} \mathrm{Ce}$ precipitação pluviométrica de $1.552 \mathrm{~mm}$.

A coleção conta com 160 plantas, das quais 60, que apresentavam frutificação no momento da seleção, foram agrupadas, preliminarmente, segundo algumas características morfológicas semelhantes, tais como: altura da planta, formato de copa, altura de inserção do primeiro ramo e coloração da folha (definida através da carta de cores de Munsell (1994), coletando-se o terceiro par de folhas completamente desenvolvidas, da parte mediana da copa).

Com auxílio da régua graduada, mediu-se altura das plantas (m), diâmetro da copa $(\mathrm{m})$ e do caule $(\mathrm{cm})$ a $20 \mathrm{~cm}$ do solo, comprimento médio do entrenó e comprimento e largura de folhas. Para determinar o comprimento do entrenó, foram utilizados 4 ramos de cada planta, e para o tamanho de folhas, utilizaram-se 100 folhas por planta.

Quando os frutos atingiram a maturação, seis semanas após a antese, procedeu-se à colheita de 100 frutos por planta, visando a avaliar suas características físicas. Determinou-se então a massa fresca média (g) de fruto, casca e sementes. O rendimento em polpa foi obtido pela diferença entre massa do fruto casca e semente. Determinaram-se também comprimento e a largura $(\mathrm{mm})$ dos frutos, com o auxílio de um paquímetro, e o número médio de sementes por fruto.

O delineamento experimental foi inteiramente casualisado (DIC), com 4 tratamentos (quatro grupos de jabuticabeiras), 5 repetições com 2 plantas por parcela, totalizando dez plantas por tratamento. Os dados foram submetidos à análise de variância $(\mathrm{F})$ e as médias comparadas pelo teste de Tukey, quando apresentaram significância.

\section{RESULTADOS E DISCUSSÃO}

Segundo suas características morfológicas, as plantas foram agrupadas em JAB 01, JAB 02, JAB 03 e JAB 04, conforme descrição detalhada de cada grupo (Tabela 1 ).

JAB 01- Plantas com copa de formato ovóide e muito frondosa. A folhagem é compacta e densa, ramos dispostos na forma de taça aberta, tronco principal com muitas ramificações, emissão de ramos secundários a partir de $20 \mathrm{~cm}$ do solo e folhas extremamente grandes e lanceoladas (Figura 1a).

JAB 02 - Plantas com copa de formato esférico, pequena e muito ramificada, folhagem esparsa e pouco densa, ramos dispostos na forma de taça um tanto fechada, tronco principal com muitas ramificações, emissão de ramos secundários a partir de $50 \mathrm{~cm}$ do solo e folhas extremamente pequenas (Figura $1 b$ ).

JAB 03 - Plantas com copa de formato esférico, folhagem compacta, ramos dispostos na forma de taça, tronco principal com poucas ramificações, emissão de ramos secundários a partir de $50 \mathrm{~cm}$ do solo e folhas pequenas (Figura 1c)

JAB 04 - Plantas com copa de formato umbeliforme, pequena e muito ramificada, folhagem compacta e densa, ramos dipostos na forma de taça aberta, tronco principal com muitas ramificações, emissão de ramos secundários a partir do colo e folhas lanceoladas mais largas (Figura 1d).

Os dados das características morfológicas das plantas são apresentados na Tabela 2. A análise de altura de planta demonstrou que o grupo JAB 04 foi o de menor porte $(3,4 \mathrm{~m})$, diferindo estatisticamente dos outros grupos, além de apresentar produção precoce, fato extremamente desejável, considerando-se a necessidade de produzir em período fora da época de maior oferta da fruta, o que possibilita obtenção de melhores preços no mercado.

De acordo com Donadio et al. (2002), para muitas frutíferas, o tamanho da copa pode ser controlado ou até reduzido com uso de poda, porta-enxerto e até mesmo com uso de reguladores de crescimento, possibilitando realizar o adensamento no plantio. A caracterização e a identificação de baixa estatura, como observado no grupo JAB 04, têm importância em programas de melhoramento genético visando à redução de porte para recomendação em plantios adensados. Os autores afirmam ainda que, para jabuticabeira 'Sabará', o porte médio atingido na faixa etária de 20 anos, em Jaboticabal, foi de 5,33 m de altura, valores similares aos das plantas utilizadas no presente estudo.

TABELA 1 - Agrupamento preliminar por características semelhantes, de plantas de jabuticabeiras, pertencentes à coleção da FCAV/UNESP, JaboticabalSP, 2003.

\begin{tabular}{|c|c|c|c|c|}
\hline Grupo & Formato da Copa $^{1}$ & Altura & Coloração Folha $^{2}$ & Altura de Inserção do $1^{\circ}$ Ramo \\
\hline JAB 01 & Ovóide & Intermediária & $4 / 2$ & Intermediária (Média - Baixa) \\
\hline JAB 02 & Esférica & Alta & $6 / 1$ & Alta \\
\hline JAB 03 & Esférica & Intermediária & $6 / 2$ & Média \\
\hline JAB 04 & Umbeliforme & Baixa & $5 / 2$ & Baixa \\
\hline
\end{tabular}

${ }^{1}$ Citado por Ramalho (1975); ${ }^{2}$ Segundo Munsell Soil Color(1994).

TABELA 2 - Características morfológicas de 4 grupos de jabuticabeiras, pertencentes à coleção da FCAV/UNESP, Jaboticabal - SP, 2003.

\begin{tabular}{lcccccc}
\hline Grupos & Altura Planta $(\mathrm{m})$ & $\begin{array}{c}\text { Diâmetro Caule } \\
(\mathrm{cm})\end{array}$ & $\begin{array}{c}\text { Diâmetro Copa } \\
(\mathrm{m})\end{array}$ & $\begin{array}{c}\text { Largura Folhas } \\
(\mathrm{cm})\end{array}$ & $\begin{array}{c}\text { Comprimento Folhas } \\
(\mathrm{cm})\end{array}$ & $\begin{array}{c}\text { Comprimento Entrenó } \\
(\mathrm{cm})\end{array}$ \\
\hline JAB 01 & $4,8 \mathrm{a}$ & $27 \mathrm{~b}$ & $4,8 \mathrm{~b}$ & $1,6 \mathrm{~b}$ & $5,6 \mathrm{a}$ & $7,3 \mathrm{a}$ \\
JAB 02 & $5,6 \mathrm{a}$ & $38 \mathrm{ab}$ & $7,0 \mathrm{a}$ & $1,6 \mathrm{~b}$ & $3,6 \mathrm{~b}$ & $2,7 \mathrm{c}$ \\
JAB 03 & $5,0 \mathrm{a}$ & $39 \mathrm{a}$ & $6,8 \mathrm{a}$ & $1,4 \mathrm{~b}$ & $3,3 \mathrm{~b}$ & $3,1 \mathrm{c}$ \\
JAB 04 & $3,4 \mathrm{~b}$ & $41 \mathrm{a}$ & $4,8 \mathrm{~b}$ & $2,3 \mathrm{a}$ & $5,8 \mathrm{a}$ & $4,6 \mathrm{~b}$ \\
\hline Média & 4,7 & 36 & 5,8 & 1,7 & 4,5 & 4,4 \\
\hline CV $(\%)$ & 15,4 & 18,1 & 9,1 & 9,2 & 5,9 & 11,5 \\
\hline
\end{tabular}

Médias seguidas pela mesma letra na coluna não diferem entre si, pelo Teste Tukey, a $5 \%$ de probabilidade. 


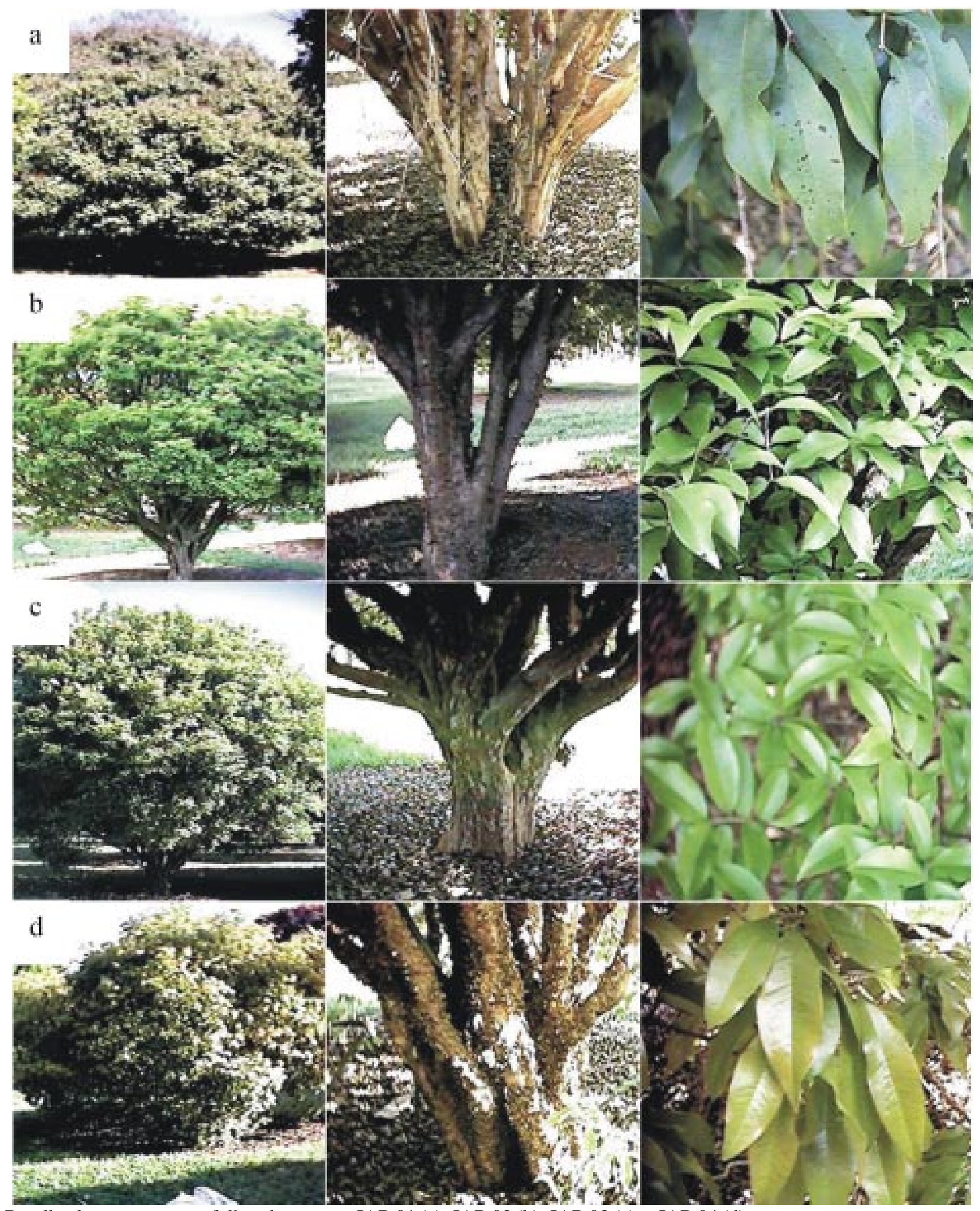

FIGURA 1- Detalhe da copa, tronco e folhas dos grupos JAB 01 (a); JAB 02 (b); JAB 03 (c), e JAB 04 (d).

Quanto ao diâmetro de caule, os resultados demonstraram que o grupo JAB 04 apresentou o maior diâmetro $(41 \mathrm{~cm})$, porém não diferindo significativamente de JAB 02 e JAB 03 e JAB 01 o menor $(27 \mathrm{~cm})$. Este valor é próximo ao citado por Lorenzi (1992), que menciona uma variação de 30 a $40 \mathrm{~cm}$ de diâmetro e superiores ao citado por Donadio (2000), de $26 \mathrm{~cm}$. Apesar de o grupo JAB 04 apresentar o maior diâmetro de caule, observa-se que possui a menor altura de planta $(3,4 \mathrm{~m})$.

Observou-se diferença significativa para diâmetro de copa, sendo que os grupos JAB 02 e JAB 03 tiveram os maiores resultados, 7,0 e $6,8 \mathrm{~m}$, respectivamente, enquanto JAB 01 e JAB 04 apresentaram os menores valores $(4,8 \mathrm{~m})$, isto quando comparamos com o valor citado por Donadio et al. (2002), de 5,7 m. Estes resultados permitem separar o material em estudo em dois grupos: JAB 02 e JAB 03 (com maior diâmetro de copa) e JAB 01 e JAB 04 (com menor diâmetro).

Os grupos JAB 01 e JAB 04, quanto ao comprimento de folhas, não apresentaram diferença significativa, sendo estes valores maiores que os observados para os grupos JAB 02 e JAB 03 (Tabela 2), variação esta superior à citada por Donadio (2000), que foi de 2,4 a 4,3 cm. Para a largura da folha, as plantas do grupo JAB $04(2,3 \mathrm{~cm})$ se aproximaram do valor citado por Lorenzi (1992), de $2 \mathrm{~cm}$ a $3 \mathrm{~cm}$, resultados superiores aos demais grupos.

Quanto ao comprimento de entrenó, JAB 01(7,3 cm) destacouse significativamente das demais ,e JAB $04(4,6 \mathrm{~cm})$ apresentou um valor intermediário que, por sua vez, foi maior que o dos grupos JAB $02(2,7 \mathrm{~cm})$ e JAB $03(3,1 \mathrm{~cm})$, que não diferiram entre si.

As características físicas dos frutos são apresentadas na Tabela 3. A massa fresca média dos frutos variou de 1,6 a 4,5g, ou seja, alguns dos grupos estudados apresentaram frutos duas vezes mais pesados, destacando-se os de JAB 01 e JAB 02, com 4,5 e 4,3g, respectivamente, diferindo significativamente do grupo JAB $04(1,6 \mathrm{~g})$, indicando a 
diversidade do material em estudo. Barros et al. (1996) encontraram valores de 4,0g, aproximando-se dos observados para os grupos JAB 01 e JAB 02. Entretanto, Pereira et al. (2000) verificaram valores que variaram de 7 a $15 \mathrm{~g}$, diferença que se explica devido ao fato de os frutos utilizados terem crescido em condições climáticas mais favoráveis (dias longos, alta temperatura e níveis pluviométricos adequados) do que os frutos colhidos por Barros et al. (1996) e os do presente trabalho.

Para percentagem de casca, o grupo JAB 04 apresentou maior participação do total do fruto (49\%), diferindo significativamente dos grupos JAB 02 e JAB 03, enquanto os menores valores foram observados para JAB 02 (33\%). Essa característica, segundo Donadio et al. (2002), está associada não só ao tamanho do fruto, mas também à espessura da casca, que é um importante componente da resistência física do fruto, já que a jabuticaba apresenta sérios problemas quanto à perecibilidade.

Quanto ao número de sementes por fruto, nota-se que existe grande variabilidade, sendo que o maior número observado foi no grupo JAB 01, com média de 3,1 sementes por fruto; para JAB 02 e JAB 03, o número médio de sementes encontrado foi intermediário (2,2 sementes para ambos) e JAB $04(1,5)$, resultados semelhantes àqueles citados por Soubihe Sobrinho \& Gurgel (1952); Benza (1980); Pereira et al. (2000) e Donadio et al. (2002).
Quando se analisa a massa das sementes no total do fruto, temse que a menor participação é no grupo JAB 04, apresentando $13 \%$, enquanto nos demais grupos a média é de $35 \%$. Os dados de rendimento em polpa (Tabela 3) mostram que o JAB 04 se destacou pelo maior rendimento (38\%), diferindo significativamente de JAB 01 , que apresentou menor porcentual (23\%); no entanto, esses valores são inferiores aos obtidos por Pereira et al. (2000), que obtiveram uma variação de 51 a $68 \%$ e Donadio et al. (2002), com 67 a 69\%. Esta diferença, que chega a ser duas vezes inferior para o material em estudo, pode estar ligada à disponibilidade de água.

O maior valor para comprimento e largura de fruto encontrado foi para o grupo JAB 01 , sendo de 17,8 e $15,3 \mathrm{~mm}$, respectivamente, diferindo do JAB 04, que apresentou menor comprimento e largura, 13,3 e $12,1 \mathrm{~mm}$, respectivamente. Resultados semelhantes foram encontrados por Benza (1980), quando os diâmetros variaram de 10 a $35 \mathrm{~mm}$, por Donadio et al. (2002), que encontraram de 16 a $22 \mathrm{~mm}$ e por Pereira et al. (2000) de 22 a $31 \mathrm{~mm}$.

Quanto ao formato do fruto, tem-se também, como nos demais fatores, alta variabilidade, sendo que os grupos JAB 01 e JAB 04 são oblongos, para JAB 03 são arredondados e para JAB 02 são achatados. Mais estudos devem ser realizados com estes grupos.

TABELA 3 - Características físicas de fruto de quatro grupos de jabuticabeira, pertencente à coleção da FCAV/UNESP, Jaboticabal - SP, 2003.

\begin{tabular}{|c|c|c|c|c|c|c|c|c|c|}
\hline \multirow[t]{2}{*}{ Grupos } & \multicolumn{3}{|c|}{ Massa fresca média (g) } & \multicolumn{3}{|c|}{ Participação média do total no fruto (\%) } & \multirow[t]{2}{*}{$\begin{array}{l}\mathrm{N}^{\circ} \text { sementes/ } \\
\text { fruto }\end{array}$} & \multicolumn{2}{|c|}{$\begin{array}{l}\text { Diâmetro médio de fruto } \\
\qquad(\mathrm{mm})\end{array}$} \\
\hline & Fruto & Casca & Semente & Casca & Semente & Polpa & & $\bar{T}$ & $\mathrm{~L}$ \\
\hline JAB 01 & $4,5 \mathrm{a}$ & $2,0 \mathrm{a}$ & $1,4 \mathrm{a}$ & $46 \mathrm{a}$ & $31 \mathrm{a}$ & $23 \mathrm{~b}$ & $3,1 \mathrm{a}$ & $17,8 \mathrm{a}$ & $15,3 \mathrm{a}$ \\
\hline JAB 02 & $4,3 \mathrm{a}$ & $1,4 \mathrm{ab}$ & $1,5 \mathrm{a}$ & $33 \mathrm{~b}$ & $37 \mathrm{a}$ & $30 \mathrm{ab}$ & $2,2 \mathrm{~b}$ & $15,8 \mathrm{ab}$ & $16,4 \mathrm{a}$ \\
\hline JAB 03 & $3,7 \mathrm{ab}$ & $1,3 \mathrm{bc}$ & $1,3 \mathrm{a}$ & $36 \mathrm{~b}$ & $35 \mathrm{a}$ & $29 \mathrm{~b}$ & $2,2 \mathrm{~b}$ & $16,0 \mathrm{ab}$ & $15,7 \mathrm{a}$ \\
\hline JAB 04 & $1,6 \mathrm{~b}$ & $0,8 \mathrm{c}$ & $0,2 \mathrm{~b}$ & $49 \mathrm{a}$ & $13 \mathrm{~b}$ & $38 \mathrm{a}$ & $1,5 \mathrm{c}$ & $13,3 \mathrm{~b}$ & $12,1 \mathrm{~b}$ \\
\hline Média & 3,5 & 1,4 & 1,1 & 41 & 29 & 30 & 2,2 & 14,9 & 15,7 \\
\hline $\mathrm{CV}(\%)$ & 24,1 & 24,5 & 22,8 & 11,5 & 17,4 & 16,2 & 15,9 & 8,2 & 9,6 \\
\hline
\end{tabular}

* Médias seguidas pela mesma letra na coluna não diferem entre si, pelo Teste Tukey, a 5\% de probabilidade. T: transversal e L: longitudinal

\section{CONCLUSÕES}

1. Constatou-se uma grande variabilidade entre os grupos de jabuticabeira cultivada no Câmpus da FCAV-UNESP, indicando a necessidade de serem propagadas vegetativamente, para fixação de características, visando ao cultivo comercial.

2. Por apresentar menor porte, menor diâmetro de copa, maior rendimento em polpa e precocidade de produção quando comparado aos demais, o grupo JAB 04 mostra-se como material mais promissor para utilização em programas de melhoramento.

\section{REFERÊNCIAS}

ANDERSEN, O.; ANDERSEN, V. U. As Frutas Silvestres Brasileiras. Rio de Janeiro: Globo, 203p. 1989.

BAILEY, L.H. Manual de tropical and sudtropical fruits. New York: MacMillian, 1920.

BARROS, R. S.; FINGER, F.L.; MANGALHÃES, M.M. Changer in non-structural carbohydrates in developing fruit of Myrciaria jaboticaba. Scentia Horticulturae, Amsterdam, v.66, p.209-215, 1996.

BENZA, J. C. 143 Frutales Nativos. Universidade Nacional Agraria La Molina, Peru. 1980. 366p.

CORREA, M.P. Plants utilized in Brasil. Rio de Janeiro: Ministério da agricultura, 1969.

DONADIO, L. C. Jabuticaba (Myrciaria jaboticaba (Vell.) Berg). Jaboticabal: FUNEP, 2000. 55p. (Série Frutas Nativas, 3).

DONADIO, L. C.; MÔRO, F. V.; SERVIDONE, A. A. Frutas Brasileiras. Jaboticabal: FUNEP, 2002. 288p.
GOMES,R.P. Fruticultura Brasileira. 9. ed. São Paulo: Nobel, 1983. 446p.

LORENZI, H. Árvores brasileiras: manual de identificação e cultivo de plantas arbóreas nativas do Brasil. Nova Odessa: Editora Plantarum, 1992. p.267.

MANGALHÃES, M.M.; BARROS, R. S.; FINGER, F.L. Changer in nonstructural carbohydrates in developing fruit of Myrciaria jaboticaba. Scentia Horticulturae, Amsterdam, v.66, p.17-22, 1996.

MUNSELL Soil color carts, New York: Macbeth, 1994.

OCHSE, J.J.; SOULE JR,M.J.;DIJKMAN, M. J.; WEHLBURGC. Tropical and subtropical agriculture. 2. ed. New York: The Macmillan Company,1966. p. 684-686.

PÉREZ, D.M; MARTINS, A.B.G. Jaboticaba (Myrciaria cauliflora Berg.): una fruta brasilena. In: CASTILHO, J.G.C.; LIMA, A.T.(Org.). Frutales para o México. México: Casa Abierta, 2002. p. 170-188.

PEREIRA, M.C.T.; SALOMÃO, L.C.C.; MOTA, W.F.; VIEIRA, G. Atributos físicos e químicos de frutos de oito clones de jabuticabeiras. Revista Brasileira de Fruticultura, Jaboticabal, v. 22, n. Especial, p.16-21, julho, 2000.

RAMALHO, R.S. Dendrologia terminologia.Viçosa: UFV, 1975. v. 1, $123 \mathrm{p}$.

SOUBIHE SOBRINHO, J; GURGEL, J. T.A. Características das sementes de Myrtaceas frutíferas. Revista de Agricultura, Piracicaba, p. 8390, 1952.

WILBANK, M.V.; CHALFUN, N.N.J.; ANDERSEN, O.O. The jaboticaba in Brazil. Proceedings of the Americans Society for Hoticutural Science, Alexandria, v.27 A, p.57-69, 1983. 